In the levels of Magdalenian $\mathrm{v}$ and vi the climate is still cold; but the horse is less abundant. For a time the reindeer is important and then gives way to the deer. Birds become abundant. There is no very marked change in the character of the stone industry; the bone artefacts show a characteristic 'fossil' in the form of a staghorn point with a forked base; barbed harpoons appear. Objects of art become scarcer; sculpture disappears, and the art form is reduced to simple graving. Both realist and decorative art weaken and are less varied.
In the material of this level, the Azilians made their hearth. The stone industry above the Magdalenian levels resumes the forms of the Aurignacian culture with characteristic delicacy and beauty of retouch. Circular scrapers determine the Azilian character of the culture, their evidence being confirmed by the harpoons, which become more and more evolved.

With the close of this period man's occupation of the cave, save for burial purposes, comes to an end.

\title{
The British Institute of Radiology
}

\section{Annual Congress and Exhibition}

$\mathrm{T}$ HE tenth Annual Congress and Exhibition of the British Institute of Radiology was held at the Central Hall, Westminster, on December 2-4, 1936. The arrangements differed to some extent from those of previous years, notably in the inclusion of a section of the exhibition devoted to physical research. Among the institutions represented in this section were St. Bartholomew's, the Royal Cancer and the Mount Vernon Hospitals, Radium Beam Therapy Research, and the National Physical Labora. tory, while Dr. Russell Reynolds showed an X-ray cinematograph apparatus. The section showed clearly the close connexion between physical research, even modern atomic physies, and the medical applications of radiations of all types. The exhibit of radium beam therapy research was concerned with a study of induced radioactivity, the principal item being a Geiger counter connected to a cathode ray oscillograph, the screen of which is photographed on a $35 \mathrm{~mm}$. ciné film operated by a mechanism so designed that the speed of motion of the film varies logarithmically with time. By the use of this device, the film moves quickly enough to record the rapid counts of a newly activated material but slows down as the activity decays, so that considerable economy in the cost of film results.

The Royal Cancer Hospital and the Mount Vernon Hospital had exhibits dealing with $\mathrm{X}$-ray and radium dosage, their measurement and typical results. The apparatus used at the Royal Cancer Hospital to determine the gamma-ray output of radium in röntgens consists of accurately made air-wall ioniza. tion chambers connected to a Lindemann electrometer, while the Mount Vernon Hospital exhibit included a valve amplifier of the DuBridge type working in conjunction with air-wall chambers and used for exploring the fields round radium sources.

The St. Bartholomew's Hospital exhibit included a demonstration of a simple form of Geiger counter employing a neon lamp for measuring weak beams of gamma-rays and locating missing radium containors, and also a series of photographic films illustrating the paths of alpha- and beta-rays in celluloid and in photographic emulsions.

The National Physical Laboratory showed, inter alia, transparencies illustrating the apparatus used in the standardization of X-ray and gamma-ray dosemeters and in the testing of radium containers, sensitive dosemeters for the measurement of stray radiation, and various $\mathrm{X}$-ray crystal photographs dealing with the structure of teeth, electro-plating, corrosion and fatigue in metals, etc. A further exhibit consisted of a model of a periscope for observing patients undergoing treatment with very penetrating radiations. The optical system comprises eight concave mirrors, but the system is so designed that, although the light beams fall on the mirrors at approximately $45^{\circ}$ to their normals, there is negligible distortion and the image appears normal size and nearer to the observer than the object.

The X-ray cinematograph equipment shown employs an indirect method in which the image on a fluorescent screen is photographed on $35-\mathrm{mm}$. ciné film, which has an emulsion specially developed to have a high sensitivity to the light emitted by the screen. In order to reduce the strain on the apparatus and the X-ray tube, an ingenious switch is incorporated so that the tube is only excited during actual exposure. The ciné camera is of the conventional type, fitted with a special lens operating at $f 0 \cdot 8$.

The session of the Congress devoted to physical and technical papers consisted in a symposium on "The Measurement of Tonization Current". Dr. D. E. Lea described a valve amplifier for the measurement of ionization currents which shares with the Townsend compensation method the advantage that the collecting electrode of the necessary ionization chamber is always substantially at earth potential. The ionization current is passed into a condenser connected to the grid of an electrometer triode the output of which is connected to two further stages of valve amplification, with the result that, in normal settings, a change of voltage of one millivolt on the grid of the electrometer valve brings about a change of voltage of ten volts in the output of the amplifier. The variation in the output voltage is thus sufficiently great to permit the operation of suitable relays and counting mechanisms through the intermediary of a thyratron. For the measurement of weak beams of radiations the rate of variation of the output voltage can be found over part of the full scale of the output voltmeter. By suitable adjustments the sensitivity of the instrument can be varied over a wide range.

Dr. G. W. C. Kaye, Mr. W. E. T. Perry and Mr. D. E. A. Jones described a ballistic amplifier in which troubles due to slow drifts and zero unsteadiness are avoided by isolating the detecting galvanometer from the anode circuit of the valve by the use of a blocking condenser. The apparatus may be used in two ways. In the first, the grid of the valve is 
maintained at zero potential with respect to the filament by the operation of a suitable potentiometer, the balance being tested by short-circuiting the grid resistance. If the system is balanced, short-circuiting the resistance does not alter the grid potential and the (ballistic) galvanometer does not deflect. The second method depends on the fact that, with the system unbalanced, the magnitude of the ballistic throw is proportional to the ionization current and to the capacity of the blocking condenser.

$\mathrm{Mr}$. T. B. Lane described a radiation integrator designed to measure the total quantity of radiation used in a given experiment. An ionization chamber is connected to the grid of an ordinary radio valve, in the output of which a thyratron is connected. The grid of the amplifying valve is given a considerable negative bias, which is reduced by the action of the ionization current until the valve operates the thyratron, which in turn operates a counting mechanism recording the number of units of charge liberated in the ionization chamber.

In closing the discussion, Prof. J. A. Crowther said he was inclined to doubt whether the various more or less elaborate amplifier and counting mechanisms in regular use to-day really do offer advantages over the older types of electrometer, which are inexpensive and reliable, and only have the disadvantage that their use requires some experimental skill.

The seventeenth Mackenzie Davidson Memorial Lecture was delivered by Dr. J. D. Cockeroft, on the subject of "High velocity positive ions. Their application to the transmutation of atomic nuclei and the production of artificial radioactivity". Dr. Cockcroft dealt with this subject in his usual lucid and fascinating manner, describing the early work on atomic transformations carried out with alpha. particles, the later work with canal rays, accelerated by potential differences of many hundreds of kilovolts, produced perhaps by the Van de Graaff static machine or the 'cyclotron' of Lawrence. Pointing out how, while earlier experiments only succeeded in transmuting light elements, a large number of heavy elements have now been transmuted, and in particular bismuth has been changed into radium $\mathbf{E}$, having the same radioactive properties as the naturally occurring element. As an example of the biological significance of the effect, he mentioned the work of Hevesy, who, by injecting into plants and animals mixtures of ordinary phosphorus and radiophosphorus, has been able, by means of Geiger counters and otherwise, to detect the movements of the phosphorus in the plant or animal and explore with some accuracy the distribution of the element at any time.

\section{Educational Topics and Events}

Cambridaz.-Miss H. G. Wanklyn (Girton College) has been appointed University lecturer in the Depart. ment of Geography and Dr. S. R. Nockolds (Trinity College) University demonstrator in mineralogy and petrology.

OXFORD.-The electors to Dr. Lee's professorship of chemistry intend to proceed to the election of a successor to Prof. Frederick Soddy on January 16, 1937. It is the desire of the sub-faculty of chemistry that, if possible, a physical chemist be appointed. To this end some changes in the statute defining the duties of the new professor were proposed in Congre- gation on December 1 and after discussion carried. The main difficulty of the new proposal, brought out in the discussion, is that while the new professor is to be given the direction of both the Inorganic and the Physical Chemistry Departments, there is, in fact, at present no official University department of physical chemistry, the whole of the teaching, and much of the research, in this subject being done in college laboratories.

It is now settled that Lord Nuffield's benefaction of $£ 2,000,000$ to the medical school is to be paid in thirteen equal half-yearly instalments for the next six and a half years. Mr. W. M. Goodenough of the Radcliffe Hospital and Barclays Bank has been appointed chairman of the Trustees.

THE twenty-fifth Annual Conference of Educational Associations will be held at University College, London, W.C.1, on January 4-11, under the presidency of the Right Hon. W. Ormsby-Gore, whose presidential address entitled "Some Educational Problems of our Colonial Empire" will be delivered on January 5 at 5 p.m. On January 7 at 5 p.m., a joint conference on "Problems of Education within the Empire" will be held, when the speakers will include Sir Firoz Khan Moon, the High Commissioner for India, formerly Minister for Education in the Punjab; Dr. S. F. N. Gie, South African Minister in Berlin, formerly Secretary for Education, Union of South Africa; ; Prof. G. V. Portus, professor of political science and history, University of Adelaide ; Prof. C. A. Krug, professor of education, Mount Allison University, New Brunswick; Prof. F "Clarke, director, Institute of Education, University of London. Further information can be obtained from the Conference Secretary, 29 Gordon Square, London, W.C.1.

The Annual Conference of the Geographical Association will be held in the London School of Economics, Houghton Street, Aldwych, London, W.C.2, on January 5-7, under the presidency of Sir Josiah Stamp, whose address, "Geography and Fconomic Theory", will be delivered on January 7 . A symposium on "Whither Population" will be held, when Prof. C. B. Fawcett, Dr. R. R. Kuczynski and Sir William Beveridge, among others, will speak. Among the lectures to be delivered will be one entitled "Problems of the North-Fast Coast", by G. H. J. Daysh, and one of the discussions announced is "Broadcast Geography Lessons" in which $\mathrm{L}$. Brooks, Dr. H. Thomas, and others will speak. Further information can be obtained from the Secretary, Geographical Association, c/o Municipal High School of Commerce, Princess Street, Manchester, 1.

\section{Science News a Century Ago}

Lord John Russell and the University of London

The Times of December 13, 1836, contained a copy of the Charter of the University of London signed on November 28, together with the letter of Lord John Russell to the Earl of Burlington, dated December 1, and the Earl's reply dated December 9 .

In his letter, Lord John Russell said: "I have the honour to transmit to your Lordship the charter of the University of London. 